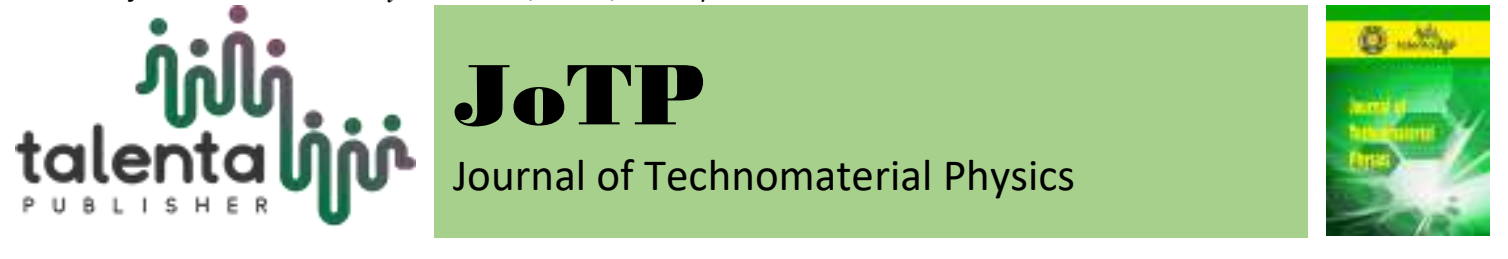

\title{
Management of Electric Field under the Sag of High Voltage Overhead Transmission Lines
}

\author{
Mester Sitepu ${ }^{1}$ and Martha Rianna ${ }^{2 *}$ \\ 1,2 Department of Physics, Faculty of Mathematics and Natural Science, Universitas Sumatera Utara \\ 20155, Indonesia
}

\begin{abstract}
There have been many methods used to determine the electric field strength under the $150 \mathrm{KV}$ overhead transmission lines (OHTL). Since it is simple and can be used for a homogeneous and multilayer medium, the method chosen for modeling the electric field under the OHTL is the complex image method (CIM). The results obtained show that the electric field strength obtained by the CIM is in agreement with that obtained by Complex Determine Method (CDM). Furthermore, the results obtained from the CIM model fit well with the field data. The OHTL height that must be raised so that the electric field strength under the OHTL will meet the WHO quality standards is $3 \mathrm{~m}$ at location A and $2 \mathrm{~m}$ at location $\mathrm{B}$. These results show that modelling the electric field under the OHTL using CIM is exellent.
\end{abstract}

Keyword: electric field, complex image, skin depth, electric resistivity, overhead transmission lines and sag

Received 04 November 2019| Revised [02 December 2019] | Accepted [14 February 2020]

\section{Introduction}

The results of environmental monitoring, in particular the electric field strength, under the sag of $150 \mathrm{KV}$ OHTL show that the electric field strength is not only influenced by the parameters of the OHTL, but also influenced by the physical properties of the medium under the OHTL. This evidence is shown by the electric field measurements conducted in which at the same segments but different sag location, although the height of OHTL and the measurement time are relatively the same, the electric field strength obtained can be different significantly. This evidence is repeated in several monitoring occations (i.e. 2008, 2009, 2011 and 2013).

The electric field is significantly affected by the surrounding environment such as trees and buildings. But the results of measurements made on open land and paddy fields also found a very noticeable difference. Since the OHTL parameters of each segment are generally made the same, the difference of the electric field strength obtained must be caused by the physical parameters of the medium under the OHTL.

\footnotetext{
*Corresponding author at: Jl. Bioteknologi No.1 Kampus USU, Medan, Indonesia, 20155

E-mail address: martharianna@usu.ac.id
} 
In terms of reducing the electric field strength under the $150 \mathrm{KV} \mathrm{OHTL}$, there are several methods available. These methods include raising the height of OHTL from the surface such as adding new poles or strengthening the OHTL cable tension, doubling the OHTL cable [1], arranging the configuration of phase [2], installing surge protectors [3], increasing the size of cable [4] and narrowing the gap between cables of phases [5].

There are many methods that have been developed to determine the electric field strength under the OHTL. Among them are the quasi-static method [6], the classic image method [7-9], the vector magnetic potential method [10], the impedance characteristic equation method [11], the CDM method [12], the Biot Savart method [1], the integral equations method [13], the surface integral equation method [14], the 3D integration method [15], the surface voltage gradient method [16] and the finite element method [13]. However, the currently available methods of calculating the electric field strength under the $150 \mathrm{KV}$ OHTL do not accomodate the layered medium under the OHTL.

One method that uses the physical parameters of medium in determining the electric field strength is the method of classic image. Classic image method has been used for long time in exploration. Maeda [17] and Roman [18] are among the early researchers that are using the classic image method. Classic image method is a method that uses the physical parameters of subsurface medium [7]. But in the classic image method, the medium under the OHTL is assumed as homogenous and the mirrors producing image located at the boundary between the air and the medium under the OHTL. In reality, the ground consists of multilayer medium. Thus, this method cannot represent the actual medium under the OHTL.

In 1952, Yost claimed that the CIM may be the best approach that can be made to obtain the comprehensive solution of the problem. However, contribution of the CIM on the development of exploration is begun in 1970, when Bannister determined the effect of electromagnetic coupling between horizontal cables that are relatively high above the ground. In 1973, then Bannister further developed the use of CIM to determine the electromagnetic coupling effect of the OHTL which its returns are considered through the homogeneous earth. Thomson and Weaver [10] and Bannister [19] even said that the CIM can be used for electromagnetic induction of interest by researchers.

Since then there are many researchers have used CIM for processing of the field data. Among them are using CIM to determine the electric and magnetic fields strength caused by an auroral electrojet [20], caused by a wide electrojet [21], caused by the line current [22], and using the CIM to solve the vector potential Hertz, to eliminating the mutual impedance between the transmitter and the receiver on the induced polarization method and on the dipole dipole method and then to eliminate the topographic effect on the induced polarization method. 
Since there are many successful uses of CIM in various areas and situations then the use of CIM in determining the electric field strength under the $150 \mathrm{KV}$ OHTL should not encounter any problem.

\section{Materials and Methods}

Using the concept of complex image means to make the problem simpler. Furthermore, the use of CIM allows to directly obtaining the total electric field strength above the medium conductor without solving differential equations associated with the field strength vector and using the relevant boundary conditions. Thomson and Weaver [11] stated that as long as the depth of image is smaller than the characteristic length of the field strength (i.e., $\mathrm{Ri}>\delta$ ), CIM can be used to determine the total electromagnetic field strength. Bannister [23] further stated that the CIM can be used to determine the solutions of the field strength component that valid to any distance and any source. However, the theory of complex image seems forgotten by most researchers [24]. Wait and Spies [24] stated that in the case of low-frequency electromagnetic field, normally a person would not think about the theory of image. They further claimed that in the near field region (quasi-near), in the air, field strength resulting from induced currents in the medium can be presented clearly by using CIM. At low frequencies, the displacement currents are much smaller than the conduction current, thus the quasistatic field approach (quasi-static) is used more often. Therefore, determination of the electric field strength caused by the OHTL, should be easier through the CIM by means of quasistatic approach.

In general form, the electric field strength radiated by a Hertzian dipole can be given as [25]:

$$
E=-\gamma^{2} \Pi+\nabla(\nabla . \Pi)
$$

with $\nabla$ is del operator, is wave propagation constant, and $\Pi=k \Pi_{x}+l \Pi_{y}+m \Pi_{z}$ is Hertz vector potential. Note that $k, l$ and $m$ are unit vectors in $x, y$ and $z$ direction, $j=\sqrt{-1}$ is complex number, and $\gamma=\sqrt{\mu \varepsilon \omega^{2}+j \mu \omega \sigma}, \sigma, \varepsilon, \mu$ and $\omega$ are electric conductivity, electric permittivity, magnetic permeability and angular frequency respectively.

From equation (1), by assuming Hertzian dipole orientation coincide with $\mathrm{x}$ direction then $\Pi_{y}=0$. For Cartesian coordinate system, the electric field strength in $\mathrm{y}$ and $\mathrm{z}$ direction is [25]:

$$
\begin{gathered}
E_{y}=\frac{\partial}{\partial y}\left(\frac{\partial}{\partial x} \Pi_{x}+\frac{\partial}{\partial z} \Pi_{z}\right) \\
E_{z}=-\gamma^{2} \Pi_{z}+\frac{\partial}{\partial z}\left(\frac{\partial}{\partial x} \Pi_{x}+\frac{\partial}{\partial z} \Pi_{z}\right)
\end{gathered}
$$

It is clear that once the Hertz vector potential in $\mathrm{x}$ and $\mathrm{z}$ directions are obtained then the following step towards getting the electric field strength will be the same for all methods. Equations (2) and (3) show that the $\mathrm{E}_{\mathrm{y}}$ and $\mathrm{E}_{\mathrm{z}}$ components of the electric field strength can be obtained from vector potential Hertz $\Pi_{\mathrm{x}}$ and $\Pi_{\mathrm{z}}$. Vector potential Hertz $\Pi_{\mathrm{x}}$ and $\Pi_{\mathrm{z}}$ can be obtained from [6]: 


$$
\begin{gathered}
\Pi_{x}=C\left(\frac{e^{-\gamma_{0} R}}{R}-\frac{e^{-\gamma_{0} R} l}{R_{l}}\right) \\
\Pi_{z}=C \frac{x}{\rho^{2}}\left(\frac{z e^{-\gamma_{0} R}}{R}-\frac{(z+d) e^{-\gamma_{0} R_{l}}}{R_{l}}\right)
\end{gathered}
$$

Where $\rho=\sqrt{x^{2}+y^{2}},=\sqrt{z^{2}+\rho^{2}}$ is distance between the source and receiver, $R_{l}=$ $\sqrt{(z+d)^{2}+\rho^{2}}$ is distance between the image and receiver, $\gamma_{0}^{2}=\varepsilon_{0} \mu_{0} \omega^{2}$ is the propagation constant and $C=\frac{I d s}{4 \pi j \varepsilon_{0} \omega}$.

The concept of complex image is the assumption that a medium, either homogeneous or layered, can be represented by a medium of perfect electrical conductivity of infinite thickness, and the distance of its surface from the interface is $d / 2$ with $d=(1-i) \delta_{1} \beta_{1}$ is the distance between the source and its image, $i=\sqrt{-1}$ is the complex number, $\delta_{1}$ is thickness of the skin (skin depth) of the first layer and $\beta_{1}$ is the factor of the layered medium. Factors layered look can be obtained following Thomson and Weaver (1975):

Start with $\beta_{n}=1$, and assume that $k=n, n-1, \ldots, 2,1$

$$
\begin{gathered}
\beta_{k}=1 ; \gamma_{k}=\frac{\beta_{k}-\left(\sigma_{k} / \sigma_{k-1}\right)^{1 / 2}}{\beta_{k}+\left(\sigma_{k} / \sigma_{k-1}\right)^{1 / 2}} e^{\frac{-2(1+j) h_{k-1}}{\delta_{k-1}}} \\
\beta_{2}=\frac{\left(1+\gamma_{3}\right)}{\left(1-\gamma_{3}\right)} ; \gamma_{2}=\frac{\beta_{2}-\left(\sigma_{2} / \sigma_{1}\right)^{1 / 2}}{\beta_{2}+\left(\sigma_{2} / \sigma_{1}\right)^{1 / 2}} e^{\frac{-2(1+j) h_{1}}{\delta_{1}}} \\
\beta_{1}=\frac{\left(1+\gamma_{2}\right)}{\left(1-\gamma_{2}\right)} \text {, and } \delta_{1}=\sqrt{\frac{2}{\mu \omega \sigma_{1}}} \\
d=(1-j) \delta_{1} \beta_{1}
\end{gathered}
$$

In general the concept of Complex Image is given in Figure 1. Note that the transmission line S of height $\mathrm{h}$ above the ground will have an image $\mathrm{S}$ ' at a depth $(\mathrm{h}+\mathrm{d})$ from the surface of the ground. Note also that the mirror location is at a depth $\mathrm{d} / 2$. The electric field strength at point $\mathrm{P}$ of height $\mathrm{z}$ from the surface and the horizontal distance of $\mathrm{x}$ from $\mathrm{S}$ is the resultant of electric field strength caused by S and S'. The electric field strength at point $\mathrm{P}$ is therefore the resultant of horizontal component $\left(\mathrm{E}_{\mathrm{y}}\right)$ and vertical component $\left(\mathrm{E}_{\mathrm{z}}\right)$ of the electric field strength.

In CIM, electric field strength measured at an arbitrary point $\mathrm{P}$ is the electric field strength caused by two sources, namely S and its image S'. Since the image depth is proportional to the thickness of skin (skin depth $\delta$ ), then in a medium of high electrical conductivity, contribution of image can be large.

Based on the above reasons, this work is intended to study the feasibility of using the CIM in calculating the electric field strength under $150 \mathrm{KV}$ OHTL, and then modelling the electric field strength under $150 \mathrm{KV}$ OHTL by accomodating the OHTL parameters and physical parameter of medium under the OHTL.

To ensure that the CIM is suitable for use in determining the electric field strength under the sag of $150 \mathrm{KV}$ OHTL, the results obtained using CIM is compared with that obtained using CDM. Next, the model is used to determine the height of OHTL from the surface by appling curve 
fitting method. In a state of fit the OHTL parameters and the physical parameters of medium under the OHTL can be considered the same as the OHTL parameters and physical parameters of the medium under the OHTL obtained through model.

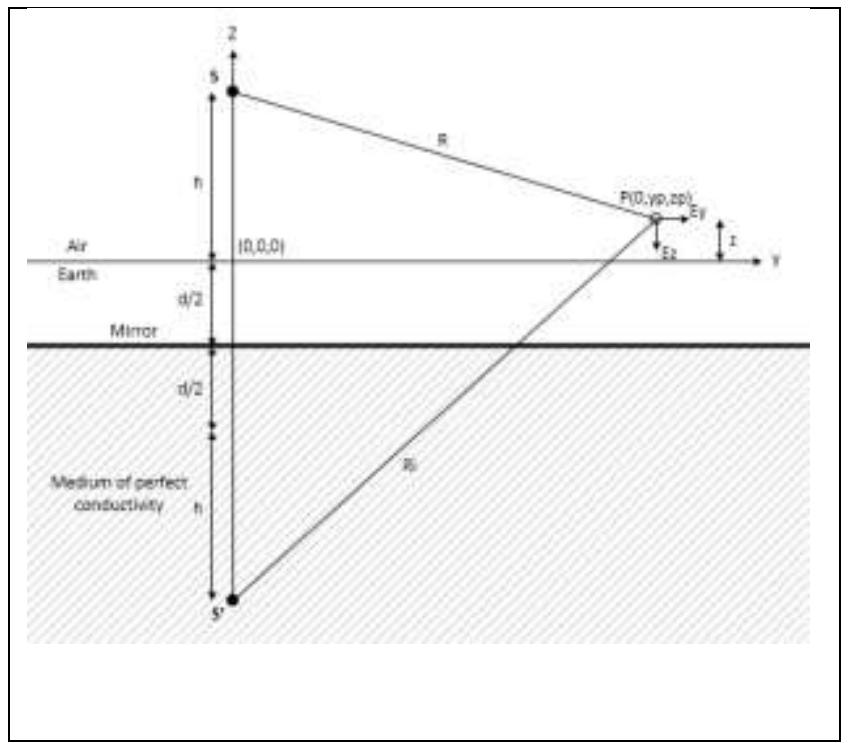

$$
\begin{aligned}
& \text { Note that: } \\
& \mathrm{P}=\text { observation point } \\
& \mathrm{S}=\text { transmission line } \\
& \mathrm{S} \text { ' }=\text { image of } \mathrm{S} \\
& \mathrm{h}=\text { height of } \mathrm{S} \text { from the surface } \\
& \mathrm{z}=\text { height of } \mathrm{P} \text { from the surface } \\
& \mathrm{x}=\text { horizontal distance between } \mathrm{S} \text { and } \mathrm{P} \\
& \mathrm{R}=\sqrt{x^{2}+(h-z)^{2}} \\
& \quad=\text { distance between } \mathrm{S} \text { and } \mathrm{P} \\
& \mathrm{R}_{\mathrm{i}}=\sqrt{x^{2}+(h+d+z)^{2}} \\
& \quad=\text { distance between } \mathrm{S}^{\prime} \text { and } \mathrm{P} \\
& d=(1-j) \delta_{1} \beta_{1}=\text { complex depth } \\
& \begin{aligned}
\mathrm{E}_{\mathrm{y}} & =\mathrm{y} \text { component of the electric field strength } \\
\mathrm{E}_{\mathrm{z}} & =\mathrm{z} \text { component of the electric field strength }
\end{aligned}
\end{aligned}
$$

Figure 1. The concept of complex image

The field data is acquired by using the Holaday ELF Survey Meter of Type HI-3604. To avoid interference, the selected locations were the locations that are relatively free from trees and buildings in its vicinity. By doing this the field data obtained is purely caused by OHTL parameters and the physical parameters of the medium under the OHTL. Field data is then fitted to the electric field strength generated by the model. In the state of fit, the electric field strength generated by the model is assumed as the parameters of the OHTL and the physical parameters of the medium under the OHTL. In this case the electric field strength caused by the OHTL at any height and any observation point can be obtained.

\section{Result and Discussion}

\subsection{Fesiability of Using CIM}

For the purpose of testing the feasibility of CIM in determining the electric field strength under the sag of $150 \mathrm{KV}$ OHTL, the electric field strength obtained using the CIM is compared with that obtained using CDM. The OHTL consist of 6 cable and three phases, the lowest height of OHTL is $20 \mathrm{~m}$ above the ground, the horizontal distance between the OHTL is $15 \mathrm{~m}$, vertical distance between the OHTL is $11.5 \mathrm{~m}$ and the diameter of each OHTL is $23.5 \mathrm{~mm}$. The height of measurement point is $1.4 \mathrm{~m}$ above the ground and the path length is $40 \mathrm{~m}(-20 \mathrm{~m}$ to $+20 \mathrm{~m})$ with the spacing of $2 \mathrm{~m}$. Due to the method used by Purwanto (2008) is basically using the classic image method, in order the comparison is relevant then the physical parameters of layered medium for CIM are set such that the mirror position is at ground surface. [12] This can be done by assuming that the layered medium is highly conductive. The comparison between 
the results obtained using CIM and that obtained using CDM are given in Figure 2, Figure 3 and Figure 4.

From Figure 2 it is clear that the horizontal electric field strength has two minimums. This is basically caused by the six lines that are splitted into two positions, namely three cables are at position $+7.5 \mathrm{~m}$ and the other three lines are at the position $+7.5 \mathrm{~m}$ from the centre. Position of the minimums are not directly beneath the cable position. This is due to the electric field strength at any point under the three phases OHTL is the resultant of the electric field strength due to all phases. Thus, the results obtained are in agreement with the expected result. On the other hand, the vertical component of the electric field strength have two maximums, namely when position of the measurement point is located under the OHTL position (Figure 4). This result is also in agreement with the expected result, since the vertical component of the electric field strength under the lines is theoretically maximum.

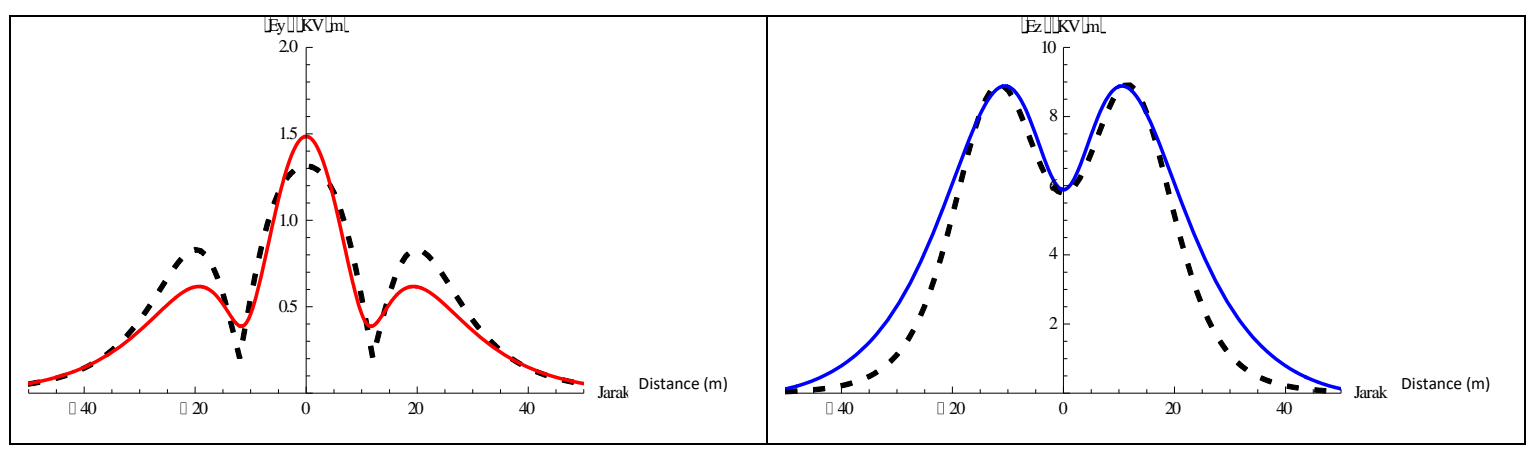

Figure 2. Comparison of $E_{y}$ as a function of $y$ distance obtained using CDM and that obtained using CIM. Note that Solid line is $\mathrm{CDM}$ and dashed line is CIM

Figure 3. Comparison of $E_{z}$ as a function of $y$ distance obtained using CDM and that obtained using CIM. Note that Solid line is CDM and dashed line is CIM

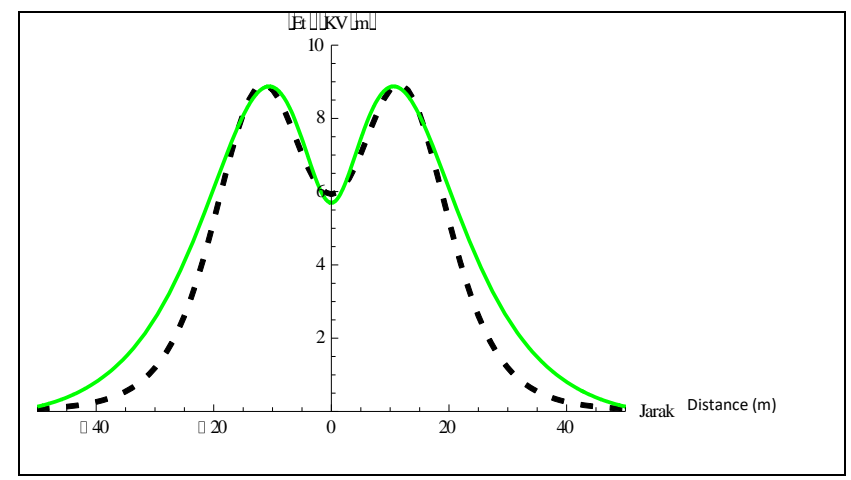

Figure 4. Comparison of $E_{t}$ as a function of $y$ distance obtained using CDM and that obtained using CIM

Note that Solid line is CDM and dashed line is CIM Results obtained by using CIM do not coincide with those obtained using CDM. The degree of similarity between the results obtaned 
by both methods however are very high. These results are indicated by the $E_{y}, E_{z}$ and $E_{t}$ obtained. The difference found here is negligible.

\subsection{Field Data Analysis}

From the comparison of results obtained using CIM with that obtained using CDM, it can be concluded that logically the CIM can be used to model the electric field strength under the sag of $150 \mathrm{KV}$ OHTL. The difference found here is possibly due to difference of the methods used. In other words, there is no fundamental difference between the two methods.

There are several sampling points gathered, however the field data analyzed here are only the field data that their electric field strengths have exceeded the quality standards according to WHO. The reaason is because the model is expected to give the height of OHTL that its generated electric field meet the WHO quality standards. Since the electric field strength at those locations have exceeded the WHO quality standards, so as not to cause any conflict, then the real locations are not exposed. To distinguish between one and other location, each of them is called location A and location B.

To get the same electric field strength between the model and the field data, the OHTL parameters and the physical parameters of medium under the OHTL are adjusted in such a way so that the sum of squared errors is minimum. The results obtained from this process are given in Figure 5 and Figure 6.

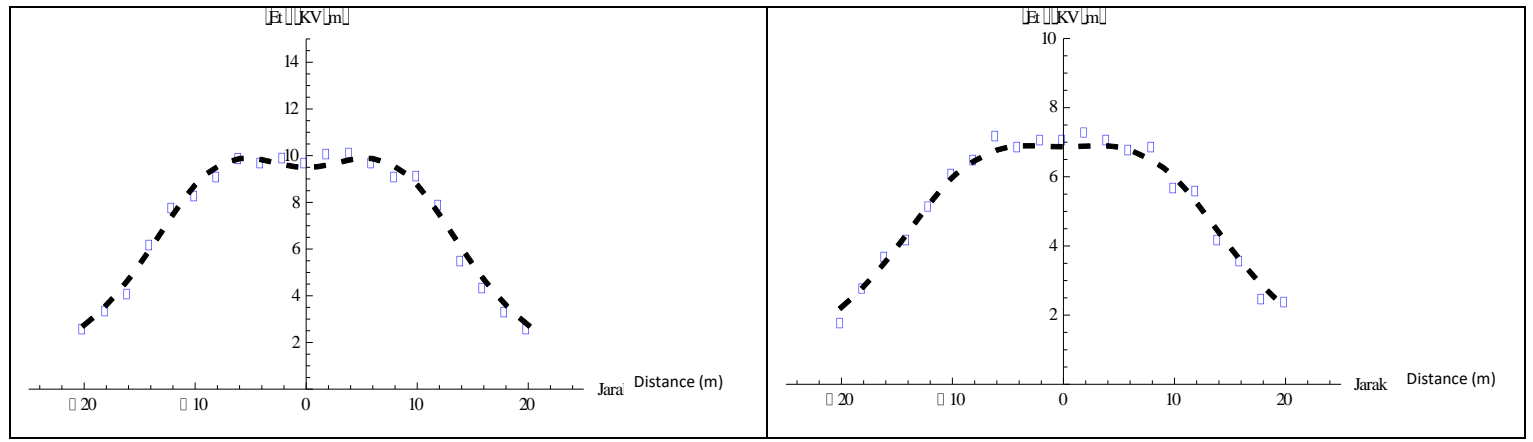

Figure 5. Fitting the field data and the electric

Figure 6. Fitting the field data and the electric field strength generated by the model for $\mathrm{E}_{\mathrm{t}}$ as field strength generated by the model for $\mathrm{E}_{\mathrm{t}}$ as a function of $\mathrm{y}$ distance (location A). Note that a function of y distance (location B). Note that dotted line is field data and dashed line is CIM dotted line is field data and dashed line is CIM

Figure 5 and Figure 6 shows that the results obtained from fitting process are exellent. The electric field strength obtained through model fits well with the field data. This result shows that the CIM is best used to model the electric field strength under the sag of $150 \mathrm{KV}$ OHTL. Errors obtained are much less than the maximum experimentation error. 
The model developed therefore is exellent to be used for determining the height of OHTL at both locations, the heights at which the electric field strength under the sag of OHTL meet the WHO quality standards. The results obtained through model show that at both locations the electric field strength generated by the OHTL are less than $5 \mathrm{KV} / \mathrm{m}$. These results are given in Figure 7 and Figure 8.

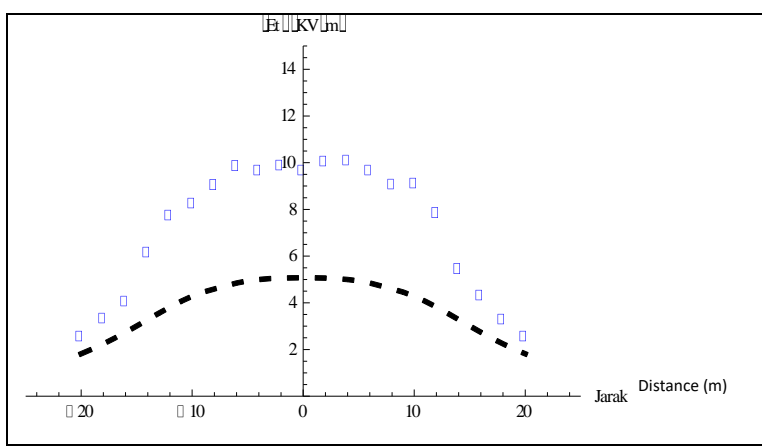

Figure 7. $\mathrm{E}_{\mathrm{t}}$ as a function of $\mathrm{y}$ distance that meet the WHO standard (location A). Note that dotted line is field data and dashed line is CIM

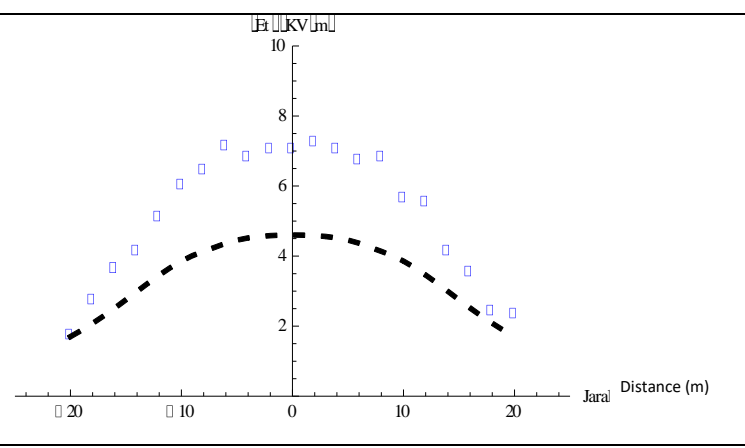

Figure 8. $\mathrm{E}_{\mathrm{t}}$ as a function of $\mathrm{y}$ distance that meet the WHO standard (location B). Note that dotted line is field data and dashed line is

CIM

From Figure 7 and Figure 8 it is clear that the electric field strength under the sag of $150 \mathrm{KV}$ OHTL will meet the WHO standard (eg. $5 \mathrm{KV} / \mathrm{m}$ ) if the height of OHTL is raised at least $3 \mathrm{~m}$ in location $\mathrm{A}$ and $2 \mathrm{~m}$ in location $\mathrm{B}$.

\section{Conclusion}

Modeling of electric field strength under the sag of $150 \mathrm{KV}$ OHTL using CIM is satisfactory. The error that occur between the results obtained using CIM and that obtained using CDM is negligible. The use of CIM in analyzing field data gives a satisfactory result. This is demonstrated by the fact that the data field fit well with the result obtained from the model. The error that occurred here is much smaller than the experimental error. The results also show that the electric field strengths under the sag of $150 \mathrm{KV}$ OHTL have already met the WHO quality standard (i.e. $5 \mathrm{KV} / \mathrm{m}$ ), if the OHTL at location A is raised at least $3 \mathrm{~m}$, while the OHTL at location $\mathrm{B}$ has to be raised at least $2 \mathrm{~m}$.

\section{Acknowledgement}

This work is supported by Universitas Sumatera Utara (USU) Medan.

\section{REFERENCES}

[1] B. Clairmont, Fundamentals of Electric and Magnetic Fields of Transmission Lines, Memphis, USA: IEEE Meeting, 2013. 
[2] M. S. H. Al Salameh and M. A. S Hassouna, "Arranging Overhead Power Transmission Line Conductors Using Swarm Intelligence Technique To Minimize Electromagnetic Fields," Progress In Electromagnetics Research B, vol. 26, pp. 213-236, 2010.

[3] X. Luwen, H. Xingzhe, L. Yongming and L. Changsheng, "Study On Shielding Optimization For Power-Frequency Electric Field Under Over Head Transmission Line", in Symposium on Radio Interference and Electromagnetic Compatibility of Substation ('08 EMI), Zhuhai, China, 2008.

[4] K. Dezelak, G. Stumberger and F. Jakl, Arrangements of Overhead Power Line Conductors Determined by Differential Evolution, Cavtat: HRO CIGRE, 2009.

[5] F. Kiessling, P. Nefzger, J. F. Nolasco, U. Kaintzyk, Overhead Power Lines Planning, Design, Construction, Berlin: Springer, 2001.

[6] R. G. Olsen and P. S. K Wong, "Characteristics of Low Frequency Electric and Magnetic Fields in the Vicinity of Electric Power Lines," IEEE Transactions on Power Delivery, vol. 7 no. 4, pp. 2046-2055, 1992.

[7] S. S. Razavipour, M. Jahangiri and H. Sadeghipoor, "Electrical Field Around the Overhead Transmission Lines," World Academy of Science, Engineering and Technology International Journal of Physical and Mathematical Sciences, vol. 6, no. 2, pp. 168-171, 2012.

[8] M. Milutinov, A. Juhas and M. Prša, "Electromagnetic Field Underneath Overhead High Voltage Power Line", in proceeding $4^{\text {th }}$ International Conference on Engineering Technologies - ICET 2009 Novi Sad, 28-30 April 2009, Thailand, 2008.

[9] M. Sitepu and Susilawati, "Solusi Vector Potensial Hertz Menggunakan Pendekatan Bayangan Komplek," Jurnal Penelitian MIPA, vol. 2, no. 1, pp. 8-16, 2008.

[10] D. J. Thomson and J.T. Weaver, "The Complex Image Approximation for Induction in A Multilayered Earth," Journal of Geophysical Research, vol. 80, no.1, pp. 123-129, 1975.

[11] A. Sofwan, Sugianto and A. Multi, "Protection Analysis Effect of Electric Field Transmission Line $500 \mathrm{KV}$ ", National Electrical Seminar, Informatics and IT's Educations. 2009.

[12] E. Purwanto, "Large Calculation of Electric Field and Magnetic Field on $500 \mathrm{KV}$ Transmission Channel and Its Influence on Human", Journal of Science and Technology, vol. 6, no. 2, 2008.

[13] P. Pao-la-or, A. Isaramongkolrak and T. Kulworawanichpong, "Finite Element Analysis of Magnetic Field Distribution for 500-kV Power Transmission Systems," Engineering Letters, vol. 18, no. 1, EL_18_1_01, 2010.

[14] O. E. Gouda, G. M. Amer and W. A. Salem, "Computational Aspects of Electromagnetic Fields Near H.V. Transmission Lines," Energy and Power Engineering, vol. 1, no.2, pp. 65-71, 2009.

[15] N. M. K. Abdel-Gawad, A. Z. El Dein and M. Magdy, "Calculation of Magnetic Field Produced by OHTL under Normal and Abnormal Conditions," WSEAS Transactions on Power Systems, vol. 9, pp. 311-318, 2014.

[16] S. Mpanga, W. Feng and C. Chun, "Electromagnetic Field Evaluation of a 500 KV High Voltage Overhead Line," Telkomnika, vol. 11 no. 2, pp. 789-796, 2013.

[17] K. Maeda, "Apparent Resistivity For Dipping Beds," Geophysics, vol. 20, no. 1, pp. 123139, 1955.

[18] I. Roman, "An Image Analysis of Multiple-layer Resistivity Problems," Geophysics, vol. 24 no. 3, pp. 485-509, 1959.

[19] P. R. Bannister, "Image Theory Results for the Mutual Impedance of Crossing Earth Return Circuits," IEEE Transactions on Electromagnetic Compatiblity, vol. 15, no. 4, pp. 158-160, 1973.

[20] R. Pirjola and A. Viljanen, "Complex Image Method for Calculating Electric and Magnetic Fields Produced by an Auroral Electrojet of Finite Length," Annales Geophysicae, vol. 16, pp. 1434-1444, 1998.

[21] D. H. Boteler, R. J. Pirjola and L. Trichtchenko, "On Calculating the Electric and Magnetic Fields Produced in Technological Systems at the Earth's Surface by a Wide Electrojet," 
Journal of Atmospheric and Solar-Terrestrial Physics, vol. 62, no. 14, pp. 1311-1315, 2000.

[22] R. J. Pirjola and D. H. Boteler, "Calculation Methods of the Electric and Magnetic Fields at the Earth's Surface Produced by a Line Current," Radio Science, vol. 37, no. 3, pp. 1-9, 2002.

[23] P. R. Bannister, "Utilization of Image Theory Techniques in Determining the Mutual Coupling Between Elevated Long Horizontal Line Sources," Radio Science, vol. 5, no. 11, pp. 1375-1381, 1970.

[24] J. R. Wait and K. P. Spies, "On the Image Representation of the Quasi Static Fields of a Line Current Source Above the Ground," Canadian Journal of Physics, vol. 47, no. 23, pp. 2731-2733, 1969.

[25] Suwitno and F. Murdiya, "Kajian Medan Magnet dan Medan Listrik pada Saluran Udara Tegangan Tinggi (SUTT) $150 \mathrm{kV}$ Kampar-Pekanbaru Berdasarkan Rekomendasi IRPA/INIRC WHO,” Jurnal Elektro ELTEK, vol. 1, no. 2, pp. 106-109, 2010. 